\title{
CONFÉRENCE
}

\section{Apport d'un nouveau substitut osseux dans la gestion parodontale des extractions pour une conservation des volumes tissulaires à visée esthétique et implantaire}

\author{
Jean-Paul Sicard \\ (Diplôme Université Bio matériaux et Systèmes implantables, Bordeaux 2) \\ jpsicardfm@me.com
}

En chirurgie pré implantaire ou péri implantaire une ostéointégration est recherchée.

La conservation d'un volume osseux est recherchée pour un résultat esthétique et fonctionnel.

De l'os peut être obtenu ou conservé par des greffes autogènes ou par régénération osseuse.

Le temps et la complexité de réalisation de ces interventions, l'allongement du traitement sont des éléments à prendre en compte.

La préservation du capital osseux ou son amélioration au cours de l'alvulsion est certainement préférable et, la mise en place de l'implant en même temps que l'extraction semble être la solution d'avenir.

La recherche de tout thérapeute est d'obtenir pour les patients des solutions les plus bénéfiques, les moins invasives, les plus efficaces, les plus rapides et les plus sûres.

Le but de l'implantologie moderne est un maintien de l'os et du tissu gingival péri implantaire à long terme : fonction et esthétique.

Le concept de «plate forme switching » et le respect de l'espace biologique semblent être garants de :

- la stabilisation à long terme autour du col de l'implant

- meilleurs résultats esthétiques

- l'amélioration de l'épaisseur des tissus mous. C'est le concept de quatre D implant position associé au «Switch plateforme » : concept moderne de l'implantologie qui demande deux à trois millimètres entre les équateurs des implants permettant à une papille inter implantaire d'exister, $1 \mathrm{~mm}$ au dessous du niveau osseux, 2 mm d'épaisseur d'os vestibulaire et linguale ou palatin protégée par trois millimètres d'épaisseur de tissu mou.

C'est la pyramide des bio matériaux thérapeutiques : système implantaire, matériaux de comblement et membrane biologique qui vont permettre aux thérapeutes de choisir une thérapeutique simplifiée.

Les comblements alvéolaires post extractionnels par des matériaux ont été décrits depuis très longtemps. Des protocoles ont été mis en place de manière reproductible permettant une amélioration clinique des résultats.

La mise en place de bio matériau dans l'alvéole amène une diminution significative de l'alvéolyse.

La technique de comblement associée à l'adjonction d'une membrane résorbable, à base de collagène évite l'envahissement du conjonctif et protège la régénération osseuse sous jacente.

Les membranes résorbables à base de collagène présentent une meilleure tolérance tissulaire que les membranes non résorbables et les résultats obtenus sont comparables avec un risque d'échec moindre pour les résorbables.

Le standard de la gestion des extractions avec mise en place ou non d'implant peut être défini comme une chirurgie avec lambeaux minimums, utilisation de bio matériaux associée avec une membrane collagénique avec suture rapprochant plus les berges.

La question que l'on peut se poser est l'utilisation de bio matériau de comblement : substituts osseux qui permettent de conserver un volume ou des produits de régénération osseuse. 
Les substituts osseux sont l'os autologue, les systèmes allo géniques (os de banque) xénogéniques (bovin, corail...) ou allo plastics (hydroxyde ha, TCP, bio verrre) ou les produits de régénération osseuse qui ont tendance à se résorber plus fortement : os autologue, matériau allo génique (os de banque) ou, matériau allo plastics (béta T C T).

La problématique des matériaux de comblement allogène ou xénogène n'ont pas de preuve formelle d'innocuité, ils n'ont pas de preuve d'absence totale de particules étrangères, ils peuvent être contaminants ou sensibilisants. Le principe de précaution du praticien ainsi que le principe de traçabilité de nos patients nous amènent à nous abstenir d'utiliser ces matériaux et à l'information incontournable des patients sur l'origine de ces bio matériaux. Les matériaux modernes de comblement semblent être les phosphates de calcium qui ont une importance biologique importante : les hydroxyapatites, l'alpha phosphate tricalcique et le béta phosphate tricalcique (matériau qui a fait sa preuve d'efficacité clinique depuis plus de 10 ans).

Le béta phosphate tricalcique avec une porosité de plus de 60 pour cent permet de piéger les cellules mésenchymateuses et hématopoïétiques nécessaires à la cicatrisation osseuse.

L'alpha phosphate tricalcique n'a pas d'intérêt clinique puisqu'en présence d'eau il se transforme en hydroxapatite compacte.

La problématique du Béta T C P est sa résorption même lente qui se termine par une résorption plus ou moins importante du volume osseux obtenu.

Fort de leur expérience les laboratoires Curasan avec leur expérience d'un Béta T C P très poreux et très stable (cérasorb) ont été capables de mettre au point deux bio matériaux synthétiques, un substitut osseux de synthèse spongieux à pores ouverts, destiné au comblement ou à la prévention des pertes de substance osseuse, c'est une hydroxyde apatite poreuse à 80 pour cent sous le nom d'osbone et ingénios.

Ce substitut osseux possède des pores ouverts et inter connectés, permettant la pénétration des vaisseaux sanguins. Sa porosité de 80 pour cent permet d'obtenir une structure osseuse spongieuse à alvéoles ouverts très proche de l'os spongieux humain.

La taille de ces pores se situe entre 250 et 450 micron $\mathrm{mm}$.

Cet hydroxyde apatite de synthèse possède une grande stabilité structurelle, qui évite la production de micro particules et, l'activité des macrophages induite par celles-ci. 